International Journal of Pure and Applied Mathematics

Volume 109 No. 1 2016, 9-28

ISSN: 1311-8080 (printed version); ISSN: 1314-3395 (on-line version)

url: http://www.ijpam.eu

doi: 10.12732/ijpam.v109i1.3

\title{
P-MOMENT EXPONENTIAL STABILITY OF DIFFERENTIAL EQUATIONS WITH RANDOM NONINSTANTANEOUS IMPULSES AND THE ERLANG DISTRIBUTION
}

\author{
R. Agarwal ${ }^{1}$, S. Hristova ${ }^{2}$, D. O'Regan ${ }^{3}$, P. Kopanov ${ }^{4}$ \\ ${ }^{1}$ Department of Mathematics \\ Texas A\&M University \\ Kingsville, Kingsville, TX 78363, USA \\ ${ }^{2,4}$ Department of Applied Mathematics and Modeling \\ Plovdiv University \\ Tzar Asen 24, 4000 Plovdiv, BULGARIA \\ ${ }^{2}$ e-mail: snehri@gmail.com \\ ${ }^{3}$ School of Mathematics, Statistics and Applied Mathematics \\ National University of Ireland \\ Galway, IRELAND
}

\begin{abstract}
In some real world phenomena a process may change instantaneously at uncertain moments and act non instantaneously on finite intervals. In modeling such processes it is necessarily to combine deterministic differential equations with random variables at the moments of impulses. The presence of randomness in the jump condition changes the solutions of differential equations significantly. The study combines methods of deterministic differential equations and probability theory. In this paper we study nonlinear differential equations subject to impulses occurring at random moments. Inspired by queuing theory and the distribution for the waiting time, we study the case of Erlang distributed random variables at the moments of impulses. The p-moment exponential stability of the trivial solution is defined and Lyapunov functions are applied to obtain sufficient conditions. Some examples are given to illustrate the results.
\end{abstract}

AMS Subject Classification: 34A37, 34F05, 34K20, 37B25

Key Words: random noninstantaneous impulses, Erlang distribution, p-moment exponential stability

Received: August 1, 2016

Published: September 1, 2016
(C) 2016 Academic Publications, Ltd. url: www.acadpubl.eu 


\section{Introduction}

In some real world phenomena a process may change instantaneously at some moments. In modeling such processes one uses impulsive differential equations (see, for example, the books [5], [6], [11] and the cited references therein). In the case when the process has instantaneous changes at uncertain moments which act non instantaneously on finite intervals one combines ideas in differential equations and probability theory. When there is uncertainty in the behavior of the state of the investigated process an appropriate model is usually a stochastic differential equation where one or more of the terms in the differential equation are stochastic processes, and this usually results with the solution being a stochastic process $([14],[15],[16],[17])$. Sometimes the impulsive action starts at an random point and remains active on a finite time interval. These type of impulses are called noninstantaneous. Recently results concerning deterministic noninstantaneous impulses were obtained for differential equations ([2], [8], [13]), delay integro-differential equations ([9]), abstract differential equations ([10]), and fractional differential equations ([1], [12]). Differential equations with instantaneously acting impulses at random times were studied in [4], [18] but there are some inaccuracies there in the mixing properties of deterministic variables and random variables, and inaccuracies in the convergence of a sequence of real numbers to a random variable.

In this paper we study nonlinear differential equations subject to impulses starting abruptly at some random points and their action continue on intervals with a given finite length. We study the case of Erlang distributed random variables defining the moments of the occurrence of the impulses. The p-moment exponential stability of the solution is studied using Lyapunov functions.

\section{Random Noninstantaneous Impulses in Differential Equations}

Let $T_{0} \geq 0$ be a given point and the increasing sequence of positive points $\left\{T_{k}\right\}_{k=1}^{\infty}$ and the sequence of nonnegative numbers $\left\{d_{i}\right\}_{i=1}^{\infty}$ be given such that $\lim _{k \rightarrow \infty} T_{k}=T \leq \infty$. Denote $d_{0}=0$.

Consider the following condition:

H1. The positive numbers $\left\{d_{k}\right\}_{k=1}^{\infty}$ are such that $\lim _{n \rightarrow \infty} \sum_{k=1}^{n} d_{k}=\infty$. 
H2. The positive numbers $\left\{d_{k}\right\}_{k=1}^{\infty}$ are such that $\lim _{n \rightarrow \infty} \sum_{k=1}^{n} d_{k}=B<$ $\infty$.

Consider the initial value problem (IVP) for the system of noninstantaneous impulsive differential equations (NIDE) with fixed points of impulses

$$
\begin{aligned}
& x^{\prime}=f(t, x(t)) \quad \text { for } \quad t \in\left(T_{k}+d_{k}, T_{k+1}\right], k=0,1,2, \ldots, \\
& x(t)=I_{k}\left(t, x\left(T_{k}-0\right)\right) \quad \text { for } t \in\left(T_{k}, T_{k}+d_{k}\right], k=1,2, \ldots, \\
& x\left(T_{0}\right)=x_{0}
\end{aligned}
$$

where $x, x_{0} \in \mathbb{R}^{n}, \quad f:[0, \infty) \times \mathbb{R}^{n} \rightarrow \mathbb{R}^{n}, \quad I_{i}:\left[T_{i}, T_{i}+d_{i}\right] \times \mathbb{R}^{n} \rightarrow \mathbb{R}^{n}$, $(i=1,2,3, \ldots)$.

Denote the solution of NIDE (1) by $x\left(t ; T_{0}, x_{0},\left\{T_{k}\right\}\right)$.

We will assume the following conditions are satisfied

H3. $f(t, 0)=0$ and $I_{k}(t, 0)=0$ for $t \geq 0, k=1,2, \ldots$.

H4. For any initial value $\left(T_{0}, x_{0}\right)$ the $O D E x^{\prime}=f(t, x)$ with $x\left(T_{0}\right)=x_{0}$ has an unique solution $x(t)=x\left(t ; T_{0}, x_{0}\right)$ defined for $t \in\left[T_{0}, P\right)$ where $P=\infty$ if condition (H1) is satisfied and $P=T+B$ is condition (H2) is satisfied.

In Section 5 we will need the following result for the initial value problem for a scalar linear differential inequality with noninstantaneous fixed moments of impulses:

$$
\begin{aligned}
& u^{\prime} \leq-m_{k} u \quad \text { for } \quad T_{k}+d_{k} \leq t \leq T_{k+1}, k=0,1,2, \ldots, \\
& u(t) \leq b_{k} u\left(T_{k}-0\right), \quad \text { for } T_{k}<t \leq T_{k}+d_{k}, k=1,2, \ldots, \\
& u\left(T_{0}\right) \leq 0
\end{aligned}
$$

Proposition 1. Let $m_{k}>0,(k=0,1,2 \ldots)$ and $b_{k}>0,(k=, 2, \ldots)$ be real constants. Then $m(t) \leq 0$ for $t \geq T_{0}$.

Proof. Let $t \in\left[T_{0}, T_{1}\right]$. Then the function $u(t)$ is continuous on $\left[T_{0}, T_{1}\right]$ and $u(t) \leq u\left(T_{0}\right) e^{-m_{0}\left(t-T_{0}\right)} \leq 0$.

Let $t \in\left(T_{1}, T_{1}+d_{1}\right]$. Then the function $u(t) \leq b_{1} u\left(T_{1}-0\right) \leq 0$.

Let $t \in\left[T_{1}+d_{1}, T_{2}\right]$. Then the function $u(t)$ is continuous on $\left[T_{1}+d_{1}, T_{2}\right]$ and $u(t) \leq u\left(T_{1}+d_{1}\right) e^{-m_{1}\left(t-T_{1}-d_{1}\right)} \leq 0$.

Continue this process.

Let the probability space $(\Omega, \mathcal{F}, P)$ be given. Let $\left\{\tau_{k}\right\}_{k=1}^{\infty}$ be a sequence of random variables defined on the sample space $\Omega$. Assume $\sum_{k=1}^{\infty} \tau_{k}=\infty$ with probability 1. 
Remark 1. The random variables $\tau_{k}$ will define the time between two consecutive impulsive moments of the impulsive differential equation with random impulses.

We will assume the following condition is satisfied

H5. The random variables $\left\{\tau_{k}\right\}_{k=1}^{\infty}, \quad \tau_{k} \in \operatorname{Erlang}\left(\alpha_{k}, \lambda\right)$ are independent with two parameters: a positive integer "shape" $\alpha_{k}$ and a positive real "rate" $\lambda$.

We will recall some properties of the Erlang distribution:

(i) If $X \in \operatorname{Erlang}\left(\alpha_{1}, \lambda\right)$ and $Y \in \operatorname{Erlang}\left(\alpha_{2}, \lambda\right)$ are independent random variables, then $X+Y \in \operatorname{Erlang}\left(\alpha_{1}+\alpha_{2}, \lambda\right)$;

(ii) The cumulative distribution function $(\mathrm{CDF})$ of $\operatorname{Erlang}(\alpha, \lambda)$ is

$$
F(x ; \alpha, \lambda)=1-e^{-\lambda x} \sum_{j=1}^{\alpha-1} \frac{(\lambda x)^{j}}{j !}=\frac{1}{(\alpha-1) !} \int_{0}^{\lambda x} y^{\alpha-1} e^{-y} d y, \quad x \geq 0
$$

and the probability density function $(\mathrm{PDF})$ is

$$
f(x ; \alpha, \lambda)=\lambda \frac{(\lambda x)^{\alpha-1}}{(\alpha-1) !} e^{-\lambda x}, \quad x>0 .
$$

Proposition 2. Let condition (H5) be satisfied and the sequence of random variables $\left\{\Xi_{k}\right\}_{k=1}^{\infty}$ be such that $\Xi_{n}=\sum_{i=1}^{n} \tau_{i}, n=1,2, \ldots$.

Then $\Xi_{n} \in \operatorname{Erlang}\left(\sum_{i=1}^{n} \alpha_{i}, \lambda\right)$.

Define the increasing sequence of random variables $\left\{\xi_{k}\right\}_{k=0}^{\infty}$ by

$$
\xi_{k}=T_{0}+\sum_{i=1}^{k} \tau_{i}+\sum_{i=1}^{k-1} d_{i}, \quad k=0,1,2, \ldots
$$

where $T_{0} \geq 0$ is a fixed point.

$$
\text { Also, } \Xi_{k}=\sum_{i=1}^{n} \tau_{i}=\xi_{k}-T_{0}-\sum_{i=1}^{k-1} d_{i}, k=1,2, \ldots
$$

Remark 2. The random variable $\xi_{n}$ will be called the waiting time and it gives the arrival time of $n$-th impulses in the impulsive differential equation with random impulses. 
Let the points $t_{k}$ be arbitrary values of the corresponding random variables $\tau_{k}, k=1,2, \ldots$. Define the increasing sequence of points

$$
T_{k}=T_{0}+\sum_{i=1}^{k} t_{i}+\sum_{i=1}^{k-1} d_{i}, \quad k=1,2, \ldots
$$

Note $T_{k}$ are values of the random variables $\xi_{k}$. The set of all solutions $x\left(t ; T_{0}, x_{0}\right.$, $\left\{T_{k}\right\}$ ) of NIDE (1) for any values $t_{k}$ of the random variables $\tau_{k}, k=1,2, \ldots$ generates a specific stochastic process with state space $\mathbb{R}^{n}$. We denote it by $x\left(t ; T_{0}, x_{0},\left\{\tau_{k}\right)\right\}$ and we will say that it is a solution of the following initial value problem for differential equations with noninstantaneous random moments of impulses (RNIDE)

$$
\begin{aligned}
& x^{\prime}(t)=f(t, x(t)) \quad \text { for } \quad t \geq T_{0}, \quad \xi_{k}+d_{k}<t<\xi_{k+1}, k=0,1, \ldots, \\
& x(t)=I_{k}\left(t, x\left(\xi_{k}\right)\right), \quad \text { for } \xi_{k}<t<\xi_{k}+d_{k}, k=1,2, \ldots \\
& x\left(T_{0}\right)=x_{0} .
\end{aligned}
$$

Definition 1. The solution $x\left(t ; T_{0}, x_{0},\left\{T_{k}\right\}\right)$ of the IVP for the IDE with fixed points of impulses (1) is called a sample path solution of the IVP for the RIDE (6).

Definition 2. A stochastic process $x\left(t ; T_{0}, x_{0},\left\{\tau_{k}\right)\right\}$ is said to be a solution of the IVP for the system of RIDE (6) if for any values $t_{k}$ of the random variable $\tau_{k}, k=1,2,3, \ldots$ and $T_{k}=T_{0}+\sum_{i=1}^{k} t_{i}, k=1,2, \ldots$ the corresponding function $x\left(t ; T_{0}, x_{0},\left\{T_{k}\right\}\right)$ is a sample path solution of the IVP for RIDE (6).

\section{Preliminary Results for Erlang Distributed Moments of Impulses}

For any $t \geq T_{0}$ consider the events

$$
\begin{gathered}
S_{0}(t)=\left\{\omega \in \Omega: t-T_{0}<\tau_{1}(\omega)\right\} \\
S_{k}(t)=\left\{\omega \in \Omega: \xi_{k}(\omega)+d_{k}<t<\xi_{k+1}(\omega)\right\}, \quad k=1,2, \ldots
\end{gathered}
$$

and

$$
W_{k}(t)=\left\{\omega \in \Omega: \xi_{k}(\omega)<t<\xi_{k}(\omega)+d_{k}\right\}, \quad k=1,2, \ldots
$$

where the random variables $\xi_{k}, k=1,2, \ldots$ are defined by (4). 
Proposition 3. For any $t \geq T_{0}$ the equality

$$
\begin{aligned}
P\left(S_{0}(t)\right) & =1-\frac{1}{\left(\alpha_{1}-1\right) !} \int_{0}^{\lambda\left(t-T_{0}\right)} y^{\alpha_{1}-1} e^{-y} d y \\
& =e^{-\lambda\left(t-T_{0}\right)} \sum_{j=1}^{\alpha_{1}-1} \frac{\left(\lambda\left(t-T_{0}\right)\right)^{j}}{j !}
\end{aligned}
$$

holds.

Corollary 1. (Upper bound of $\left.S_{0}(t)\right)$. For any $t \geq T_{0}$ the inequality

$$
P\left(S_{0}(t)\right) \leq e^{-\lambda\left(t-T_{0}\right)} \frac{\left(\lambda\left(t-T_{0}\right)\right)^{\alpha_{1}-1}}{\left(\alpha_{1}-1\right) !}
$$

holds.

Proof. We have the following

$$
\sum_{j=1}^{\alpha_{1}-1} \frac{\left(\lambda\left(t-T_{0}\right)\right)^{j}}{j !}=\frac{e^{\lambda\left(t-T_{0}\right)} \Gamma\left(\alpha_{1}, \lambda\left(t-T_{0}\right)\right)}{\left(\alpha_{1}-1\right) !}-1
$$

Apply the inequality $\Gamma(a, x) \leq \frac{x^{a} e^{-x}}{x-a-1}$ (see [7]) for the upper incomplete gamma function $\Gamma(a, x)=\int_{x}^{\infty} y^{a-1} e^{-y} d y$ and obtain

$$
\begin{aligned}
\sum_{j=1}^{\alpha_{1}-1} \frac{\left(\lambda\left(t-T_{0}\right)\right)^{j}}{j !} & \leq \frac{e^{\lambda\left(t-T_{0}\right)} \Gamma\left(\alpha_{1}, \lambda\left(t-T_{0}\right)\right)}{\left(\alpha_{1}-1\right) !}-1 \\
& \leq \frac{e^{\lambda\left(t-T_{0}\right)}}{\left(\alpha_{1}-1\right) !} \frac{\left(\lambda\left(t-T_{0}\right)\right)^{\alpha_{1}} e^{-\lambda\left(t-T_{0}\right)}}{\lambda\left(t-T_{0}\right)-\alpha_{1}-1}-1 \\
& \leq \frac{1}{\left(\alpha_{1}-1\right) !} \frac{\left(\lambda\left(t-T_{0}\right)\right)^{\alpha_{1}}}{\lambda\left(t-T_{0}\right)}-1
\end{aligned}
$$

Lemma 1. Let conditions (H1),(H5) be satisfied and $t \geq T_{0}$. 
Then the probability that there will be exactly $k$ impulses until time $t$ is

$$
P\left(S_{j}(t)\right)= \begin{cases}0, & \text { for } j>k, \\ e^{-\lambda\left(t-T_{0}-\sum_{i=1}^{j} d_{i}\right)} \sum_{m=A_{j}}^{A_{j+1}-1} \frac{\left(\lambda\left(t-T_{0}-\sum_{i=1}^{j} d_{i}\right)^{m}\right.}{m !} & \\ =\int_{0}^{\lambda\left(t-T_{0}-\sum_{i=1}^{j} d_{i}\right)}\left(\frac{y^{A_{j}-1}}{\left(A_{j}-1\right) !}-\frac{y^{A_{j+1}-1}}{\left(A_{j+1}-1\right) !}\right) e^{-y} d y, & \text { for } j \leq k,\end{cases}
$$

where $A_{j}=\sum_{i=1}^{j} \alpha_{i}$ and $T_{0}+\sum_{i=1}^{k} d_{i} \leq t<T_{0}+\sum_{i=1}^{k+1} d_{i}$.

Proof. From the definition of $S_{j}(t)$ we get

$$
\begin{aligned}
P\left(S_{j}(t)\right) & =P\left(T_{0}+\sum_{i=1}^{j} \tau_{i}+\sum_{i=1}^{j} d_{i}<t<T_{0}+\sum_{i=1}^{j+1} \tau_{i}+\sum_{i=1}^{j} d_{i}\right) \\
& =P\left(\Xi_{j}<t-T_{0}-\sum_{i=1}^{j} d_{i}\right)-P\left(\Xi_{j+1}<t-T_{0}-\sum_{i=1}^{j} d_{i}\right) .
\end{aligned}
$$

Let $j>k$. From the definition of $k$ it follows that

$$
t-T_{0}-\sum_{i=1}^{j} d_{i} \leq t-T_{0}-\sum_{i=1}^{k+1} d_{i}<0
$$

and therefore $P\left(\Xi_{j}<t-T_{0}-\sum_{i=1}^{j} d_{i}\right)=0, P\left(\Xi_{j+1}<t-T_{0}-\sum_{i=1}^{j} d_{i}\right)=0$ and $P\left(S_{j}(t)\right)=0$.

Now, let $j \leq k$. Then $t-T_{0}-\sum_{i=1}^{j} d_{i} \geq t-T_{0}-\sum_{i=1}^{k} d_{i} \geq 0$. From Proposition 2, equality (9) and formula (3) we obtain

$$
\begin{aligned}
P\left(S_{j}(t)\right)= & \sum_{m=1}^{\sum_{i=1}^{j+1} \alpha_{i}-1} \frac{\left(\lambda\left(t-T_{0}-\sum_{i=1}^{j} d_{i}\right)^{m}\right.}{m !} e^{-\lambda\left(t-T_{0}-\sum_{i=1}^{j} d_{i}\right)} \\
& -\sum_{m=1}^{\sum_{i=1}^{j} \alpha_{i}-1} \frac{\left(\lambda\left(t-T_{0}-\sum_{i=1}^{j} d_{i}\right)^{m}\right.}{m !} e^{-\lambda\left(t-T_{0}-\sum_{i=1}^{j} d_{i}\right)} .
\end{aligned}
$$


Also,

$$
\begin{aligned}
P\left(S_{j}(t)\right)= & \frac{1}{\left(A_{j}-1\right) !} \int_{0}^{\lambda\left(t-T_{0}-\sum_{i=1}^{j} d_{i}\right)} y^{A_{j}-1} e^{-y} d y \\
& -\frac{1}{\left(A_{j+1}-1\right) !} \int_{0}^{\lambda\left(t-T_{0}-\sum_{i=1}^{j} d_{i}\right)} y^{A_{j+1}-1} e^{-y} d y .
\end{aligned}
$$

Remark 3. Let conditions (H1),(H5) be satisfied and $\alpha_{j+1}=1$, i.e. $\tau_{j+1} \in \operatorname{Exp}(\alpha)$. Then the formula (8) reduces to

$$
P\left(S_{j}(t)\right)=e^{-\lambda\left(t-T_{0}-\sum_{i=1}^{j} d_{i}\right)} \frac{\left(\lambda\left(t-T_{0}-\sum_{i=1}^{j} d_{i}\right)^{A_{j}}\right.}{A_{j} !} .
$$

Remark 4. Note $\sum_{m=A_{j}}^{A_{j+1}-1} \frac{(x)^{m}}{m !}$ is a polynomial $R_{j}(x)$ of power $A_{j+1}-1$ with positive coefficients.

Corollary 2. Let conditions (H1),(H5) be satisfied with $\alpha_{i}=\alpha, i=$ $1,2, \ldots$ and $t \geq T_{0}+\sum_{i=1}^{k} d_{i}$.

Then the probability that there will be exactly $k$ impulses until time $t$ is

$$
P\left(S_{k}(t)\right)=e^{-\lambda\left(t-T_{0}-\sum_{i=1}^{k} d_{i}\right)} \sum_{j=k \alpha}^{k \alpha+(\alpha-1)} \frac{\left(\lambda\left(t-T_{0}-\sum_{i=1}^{k} d_{i}\right)^{j}\right.}{j !}
$$

Lemma 2. Let conditions (H2),(H5) be satisfied and $t \geq T_{0}$. Then the probability that there will be exactly $k$ impulses until time $t$ is

$$
P\left(S_{j}(t)\right)=\left\{\begin{array}{l}
0, \text { for } t<T_{0}+B \text { and } j>k, \\
e^{-\lambda\left(t-T_{0}-\sum_{i=1}^{j} d_{i}\right)} \sum_{m=A_{j}}^{A_{j+1}-1} \frac{\left(\lambda\left(t-T_{0}-\sum_{i=1}^{j} d_{i}\right)^{m}\right.}{m !} \\
=\int_{0}^{\lambda\left(t-T_{0}-\sum_{i=1}^{j} d_{i}\right)}\left(\frac{y^{A_{j}-1}}{\left(A_{j}-1\right) !}-\frac{y^{A_{j+1}-1}}{\left(A_{j+1}-1\right) !}\right) e^{-y} d y, \\
\text { for } t<T_{0}+B \text { and } j \leq k \text { or } t \geq T_{0}+B
\end{array}\right.
$$

where $A_{j}=\sum_{i=1}^{j} \alpha_{i}$ and $T_{0}+\sum_{i=1}^{k} d_{i} \leq t<T_{0}+\sum_{i=1}^{k+1} d_{i}$. 
Proof. The proof of the case $t<T_{0}+B$ is similar to the proof in Lemma 1.

Let $t \geq T_{0}+B$. Then for all natural number $j$ the inequality $t-T_{0}-$ $\sum_{i=1}^{j} d_{i}>t-T_{0}-\sum_{i=1}^{\infty} d_{i} \geq T-T_{0}+B \geq 0$ holds and the proof is similar to the one of Lemma 1 .

Lemma 3. (Upper bound of $S_{k}(t)$ ). Let condition (H5) and one of (H1) or (H2) be satisfied. Then for any natural number $j$ we have

$$
P\left(S_{j}(t)\right) \leq K_{j} e^{-\lambda\left(t-T_{0}-\sum_{i=1}^{j} d_{i}\right)}\left(\lambda\left(t-T_{0}\right)\right)^{A_{j}}
$$

holds where $K_{j}=\max \left\{1,\left(\lambda\left(t-T_{0}\right)\right)^{\alpha_{j+1}-1}\left(\alpha_{j+1}-1\right)\right\}$.

Proof. From equality (12), inequality

$$
\begin{aligned}
& \frac{\left(\lambda\left(t-T_{0}-\sum_{i=1}^{j} d_{i}\right)\right)^{m}}{m !} \leq \frac{\left(\lambda\left(t-T_{0}\right)\right)^{m}}{m !} \leq \frac{\left(\lambda\left(t-T_{0}\right)\right)^{A_{j+1}-1}}{m !} \\
& \quad \leq\left(\lambda\left(t-T_{0}\right)\right)^{A_{j+1}-1}
\end{aligned}
$$

and Remark 4 it follows that (13) is true.

Lemma 4. Let conditions (H1), (H5) hold. Then the probability the time $t$ is immediately after the $k$-th random impulse but not far away than $d_{k}$ from it is given by

$$
P\left(W_{j}(t)\right)= \begin{cases}0, & \text { for } j>k, \\ e^{-\lambda g_{j}} \sum_{m=1}^{A_{j}-1}\left(\frac{\left(\lambda g_{i}\right)^{m}-\left(\lambda g_{j-1}\right)^{m} e^{-\lambda d_{k}}}{m !}\right) & \\ =\frac{1}{\left(A_{j}-1\right) !} \int_{\lambda g_{j}}^{\lambda g_{j-1}} y^{A_{k}-1} e^{-y} d y, & \text { for } j \leq k,\end{cases}
$$

where $A_{j}=\sum_{i=1}^{j} \alpha_{i}, D_{j}=\sum_{i=1}^{j} d_{i}, g_{j}=t-T_{0}-D_{j}$ and $T_{0}+\sum_{i=1}^{k} d_{i} \leq t<$ $T_{0}+\sum_{i=1}^{k+1} d_{i}$ 
Proof. From the definition of $W_{j}(t)$ and the random variables $\Xi_{j}$ we get

$$
\begin{aligned}
P\left(W_{j}(t)\right) & =P\left(\xi_{j}<t<\xi_{j}+d_{k}\right) \\
& =P\left(t-T_{0}-\sum_{i=1}^{j} d_{i}<\sum_{i=1}^{j} \tau_{i}<t-T_{0}-\sum_{i=1}^{j-1} d_{i}\right) \\
& =P\left(\Xi_{j}<t-T_{0}-\sum_{i=1}^{j-1} d_{i}\right)-P\left(\Xi_{j}<t-T_{0}-\sum_{i=1}^{j} d_{i}\right) .
\end{aligned}
$$

If $j>k$ then similar to the proof in Lemma 1 we get $P\left(W_{j}(t)\right)=0$.

Now, let $j \leq k$. Then $t-T_{0}-\sum_{i=1}^{j-1} d_{i} \geq t-T_{0}-\sum_{i=1}^{j} d_{i} \geq t-T_{0}-\sum_{i=1}^{k} d_{i} \geq$ 0 . From Proposition 2 and equality (3) we obtain

$$
P\left(W_{j}(t)\right)=\sum_{m=1}^{A_{j}-1} \frac{\left(\lambda g_{j}\right)^{m}}{m !} e^{-\lambda g_{j}}-\sum_{m=1}^{A_{j}-1} \frac{\left(\lambda g_{j-1}\right)^{m}}{m !} e^{-\lambda g_{j-1}}
$$

Also,

$$
\begin{aligned}
P\left(W_{j}(t)\right) & =\frac{1}{\left(A_{j}-1\right) !} \int_{0}^{\lambda g_{j-1}} y^{A_{j}-1} e^{-y} d y-\frac{1}{\left(A_{j}-1\right) !} \int_{0}^{\lambda g_{j}} y^{A_{j}-1} e^{-y} d y \\
& =\frac{1}{\left(A_{j}-1\right) !} \int_{\lambda g_{j}}^{\lambda g_{j-1}} y^{A_{j}-1} e^{-y} d y
\end{aligned}
$$

Lemma 5. Let conditions (H2),(H5) be satisfied and $t \geq T_{0}$. Then the probability the time $t$ is immediately after the $k$-th random impulse but not far away than $d_{k}$ from it is given by

$$
P\left(W_{j}(t)\right)=\left\{\begin{array}{cc}
0 & \text { for } t<T_{0}+B \text { and } j>k \\
e^{-\lambda g_{j}} \sum_{m=1}^{A_{j}-1}\left(\frac{\left(\lambda g_{j}\right)^{m}-\left(\lambda g_{j-1}\right)^{m} e^{-\lambda d_{k}}}{m !}\right) \\
=\frac{1}{\left(A_{j}-1\right) !} \int_{\lambda g_{j}}^{\lambda g_{j-1}} y^{A_{k}-1} e^{-y} d y \\
\quad \text { for } t<T_{0}+B \text { and } j \leq k \text { or } t \geq T_{0}+B,
\end{array}\right.
$$

where $A_{j}=\sum_{i=1}^{j} \alpha_{i}, D_{j}=\sum_{i=1}^{j} d_{i}, g_{j}=t-T_{0}-D_{j}$ and $T_{0}+\sum_{i=1}^{k} d_{i} \leq t<$ $T_{0}+\sum_{i=1}^{k+1} d_{i}$. 
Lemma 6. (Upper bound of $W_{k}(t)$ ). Let condition (H5) and one of (H1) or (H2) be satisfied. Then for any natural number $j$ we have

$$
P\left(W_{j}(t)\right) \leq d_{j} e^{-\lambda\left(t-T_{0}-\sum_{i=1}^{j} d_{i}\right)} \frac{\left(\lambda\left(t-T_{0}\right)\right)^{\sum_{i=1}^{j} \alpha_{i}}}{\left(A_{j}-1\right) !\left(t-T_{0}\right)} .
$$

Proof. Using the Integral mean value Theorem and $\sum_{i=1}^{j} \alpha_{i} \geq 1$ we obtain

$$
\begin{aligned}
& \left.\int_{\lambda g_{j}}^{\lambda g_{j-1}} y^{A_{j}-1} e^{-y} d y \leq \lambda d_{j}(\lambda) g_{j-1}\right)^{\sum_{i=1}^{j} \alpha_{i}-1} e^{-\lambda g_{j}} \\
& \quad \leq \lambda d_{j}\left(\lambda\left(t-T_{0}\right)\right)^{\sum_{i=1}^{j} \alpha_{i}-1} e^{-\lambda\left(t-T_{0}-\sum_{i=1}^{j} d_{i}\right)} .
\end{aligned}
$$

\section{Linear Equations with Random Noninstantaneous Impulses}

Consider the initial value problem for a scalar linear differential equation with random noninstantaneous moments of impulses:

$$
\begin{aligned}
& u^{\prime}=-m_{k} u \quad \text { for } \quad \xi_{k}+d_{k}<t<\xi_{k+1}, k=0,1,2, \ldots, \\
& u(t)=b_{k} u\left(\xi_{k}\right), \quad \text { for } \quad \xi_{k}<t<\xi_{k}+d_{k}, k=1,2, \ldots, \\
& u\left(T_{0}\right)=u_{0},
\end{aligned}
$$

where $u_{0} \in \mathbb{R}, m_{k}>0,(k=0,1,2 \ldots)$ and $b_{k} \neq 1,(k=, 2, \ldots)$ are real constants.

Lemma 7. Let the following conditions be satisfied:

1. Condition (H5) and one of the conditions (H1) or (H2) is fulfilled.

2. $m_{i}+\lambda \geq m_{k}$ for all natural $i, k: i<k$.

Then the solution of the IVP for the scalar linear differential equation with random noninstantaneous moments of impulses (18) is

$$
u\left(t ; T_{0}, u_{0},\left\{\tau_{k}\right\}\right)=\left\{\begin{array}{c}
u_{0} e^{-\sum_{i=0}^{k-1} m_{i} \tau_{i+1}}\left(\prod_{i=1}^{k} b_{i}\right) \\
\quad \text { for } \xi_{k}<t \leq \xi_{k}+d_{k}, k=1,2, \ldots, \\
u_{0} e^{-\sum_{i=0}^{k-1} m_{i} \tau_{i+1}}\left(\prod_{i=1}^{k} b_{i}\right) e^{-m_{k}\left(t-\xi_{k}-d_{k}\right)} \\
\text { for } \xi_{k}+d_{k}<t<\xi_{k+1}, k=0,1,2, \ldots
\end{array}\right.
$$


and the expected value of the solution is

$$
\begin{aligned}
& E\left(\left|u\left(t ; T_{0}, u_{0},\left\{\tau_{k}\right\}\right)\right|\right)=\left|u_{0}\right|\left\{e^{-m_{0}\left(t-T_{0}\right)} P\left(S_{0}(t)\right)\right. \\
& +\sum_{k=1}^{\infty}\left(\prod_{i=1}^{k}\left|b_{i}\right|\left(\frac{\lambda}{m_{i-1}+\lambda}\right)^{\alpha_{i}}\right) P\left(W_{k}(t)\right) \\
& \left.\quad+\sum_{k=1}^{\infty}\left(\prod_{i=1}^{k}\left|b_{i}\right|\left(\frac{\lambda}{m_{i-1}-m_{k}+\lambda}\right)^{\alpha_{i}}\right) e^{-m_{k} g_{k}} P\left(S_{k}(t)\right)\right\} .
\end{aligned}
$$

Proof. The sample path solution of (18) is given by

$$
u\left(t ; T_{0}, u_{0},\left\{T_{k}\right\}\right)=\left\{\begin{array}{c}
u_{0} e^{-\sum_{i=0}^{k-1} m_{i}\left(T_{i+1}-T_{i}-d_{i}\right)}\left(\prod_{i=1}^{k} b_{i}\right) \\
\text { for } T_{k}<t \leq T_{k}+d_{k}, k=1,2, \ldots, \\
u_{0} e^{-\sum_{i=0}^{k-1} m_{i}\left(T_{i+1}-T_{i}-d_{i}\right)}\left(\prod_{i=1}^{k} b_{i}\right) \\
e^{-m_{k}\left(t-T_{k}-d_{k}\right)} \\
\text { for } T_{k}+d_{k}<t<T_{k+1}, k=0,1,2, \ldots
\end{array}\right.
$$

The above equality and Definition 2 establishes (19).

From formula (19) and the independence of the random variables $\tau_{k}$ we obtain

$$
\begin{aligned}
& E\left(\left|u\left(t ; T_{0}, u_{0},\left\{\tau_{k}\right\}\right)\right|\right)=\left|u_{0}\right| e^{-m_{0}\left(t-T_{0}\right)} P\left(S_{0}(t)\right) \\
& +\sum_{k=1}^{\infty}\left|u_{0}\right|\left(\prod_{i=1}^{k}\left|b_{i}\right|\right) E\left(e^{-\sum_{i=0}^{k-1} m_{i} \tau_{i+1}}\right) P\left(W_{k}(t)\right) \\
& +\sum_{k=1}^{\infty}\left|u_{0}\right|\left(\prod_{i=1}^{k}\left|b_{i}\right|\right) e^{-m_{k} g_{k}} E\left(e^{\sum_{i=1}^{k}\left(m_{k}-m_{i-1}\right) \tau_{i}}\right) P\left(S_{k}(t)\right) .
\end{aligned}
$$

Using the definition of the density function of the Erlang distribution and substituting $\left(m_{i-1}+\lambda\right) x=s$ we get

$$
\begin{aligned}
& E e^{-m_{i-1} \tau_{i}}=\int_{0}^{\infty} e^{-m_{i-1} x} \frac{\lambda^{\alpha_{i}} x^{\alpha_{i}-1} e^{-\lambda x}}{\Gamma\left(\alpha_{i}\right)} d x \\
& =\frac{1}{\left(m_{i-1}+\lambda\right)^{\alpha_{i}}} \frac{(\lambda)^{\alpha_{i}}}{\left(\alpha_{i}-1\right) !} \int_{0}^{\infty} e^{-s} s^{\alpha_{i}-1} d s=\left(\frac{\lambda}{m_{i-1}+\lambda}\right)^{\alpha_{i}}
\end{aligned}
$$


and substituting $\left(m_{i-1}-m_{k}+\lambda\right) x=s$ we get

$$
\begin{aligned}
& E e^{\left(m_{k}-m_{i-1}\right) \tau_{i}}=\int_{0}^{\infty} e^{\left(m_{k}-m_{i-1}\right) x} \frac{\lambda^{\alpha_{i}} x^{\alpha_{i}-1} e^{-\lambda x}}{\Gamma\left(\alpha_{i}\right)} d x \\
& =\frac{1}{\left(m_{i-1}-m_{k}+\lambda\right)^{\alpha_{i}}} \frac{(\lambda)^{\alpha_{i}}}{\left(\alpha_{i}-1\right) !} \int_{0}^{\infty} e^{-s} s^{\alpha_{i}-1} d s \\
& =\left(\frac{\lambda}{m_{i-1}-m_{k}+\lambda}\right)^{\alpha_{i}} .
\end{aligned}
$$

Therefore,

$$
E\left(e^{\sum_{i=1}^{k}\left(m_{k}-m_{i-1}\right) \tau_{i}}\right)=\prod_{i=1}^{k}\left(\frac{\lambda}{m_{i-1}-m_{k}+\lambda}\right)^{\alpha_{i}}
$$

Substitute (22) and (23) in (21) and obtain (20).

Corollary 3. Let the conditions of Lemma 7 be satisfied with $m_{k}=$ $m, k=1,2, \ldots$ Then for any $t \geq T_{0}$

$$
\begin{gathered}
E\left(\left|u\left(t ; T_{0}, u_{0},\left\{\tau_{k}\right\}\right)\right|\right)=\left|u_{0}\right| e^{-m\left(t-T_{0}\right)} P\left(S_{0}(t)\right) \\
+\left|u_{0}\right| \sum_{k=1}^{\infty}\left(\prod_{i=1}^{k}\left|b_{i}\right|\left(\frac{\lambda}{m+\lambda}\right)^{\alpha_{i}}\right) P\left(W_{k}(t)\right) \\
+\left|u_{0}\right| e^{-m\left(t-T_{0}\right)} \sum_{k=1}^{\infty}\left(\prod_{i=1}^{k}\left|b_{i}\right| e^{m d_{i}}\right) P\left(S_{k}(t)\right)
\end{gathered}
$$

Lemma 8. (Upper bound of the expected value). Let the conditions of Lemma 7 be satisfied.

Then

$$
\begin{aligned}
& E\left(\left|u\left(t ; T_{0}, u_{0},\left\{\tau_{k}\right\}\right)\right|\right)=\left|u_{0}\right| e^{-\lambda\left(t-T_{0}\right)} \\
& \times\left\{e^{-m_{0}\left(t-T_{0}\right)} \frac{\left(\lambda\left(t-T_{0}\right)\right)^{\alpha_{1}-1}}{\left(\alpha_{1}-1\right) !}\right. \\
& \quad+\sum_{k=1}^{\infty}\left(\prod_{i=1}^{k}\left|b_{i}\right| e^{\lambda d_{i}}\left(\frac{\lambda^{2}\left(t-T_{0}\right)}{m_{i-1}-m_{k}+\lambda}\right)^{\alpha_{i}}\right) \\
& \left.\times\left(K_{k} e^{-m_{k}\left(t-T_{0}\right)}+\frac{d_{k}}{t-T_{0}}\right)\right\} .
\end{aligned}
$$


Proof. According to equality(20), Corollary 1, Lemma 3 and Lemma 6 we obtain

$$
\begin{aligned}
& E\left(\left|u\left(t ; T_{0}, u_{0},\left\{\tau_{k}\right\}\right)\right|\right) \leq\left|u_{0}\right|\left\{e^{-m_{0}\left(t-T_{0}\right)} e^{-\lambda\left(t-T_{0}\right)} \frac{\left(\lambda\left(t-T_{0}\right)\right)^{\alpha_{1}-1}}{\left(\alpha_{1}-1\right) !}\right. \\
& \quad+\sum_{k=1}^{\infty}\left(\prod_{i=1}^{k}\left|b_{i}\right|\left(\frac{\lambda}{m_{i-1}+\lambda}\right)^{\alpha_{i}}\right) d_{k} e^{-\lambda\left(t-T_{0}-\sum_{i=1}^{k} d_{i}\right)} \frac{\left(\lambda\left(t-T_{0}\right)\right)^{\sum_{i=1}^{k} \alpha_{i}}}{\left(A_{k}-1\right) !\left(t-T_{0}\right)} \\
& \left.\quad+\sum_{k=1}^{\infty}\left(\prod_{i=1}^{k}\left|b_{i}\right|\left(\frac{\lambda}{m_{i-1}-m_{k}+\lambda}\right)^{\alpha_{i}}\right) e^{-\left(m_{k}+\lambda\right) g_{k}} K_{k}\left(\lambda\left(t-T_{0}\right)\right)^{A_{k}}\right\} \\
& \leq\left|u_{0}\right| e^{-\lambda\left(t-T_{0}\right)}\left\{e^{-m_{0}\left(t-T_{0}\right)} \frac{\left(\lambda\left(t-T_{0}\right)\right)^{\alpha_{1}-1}}{\left(\alpha_{1}-1\right) !}\right. \\
& \quad+\sum_{k=1}^{\infty}\left(\prod_{i=1}^{k}\left|b_{i}\right| e^{\lambda d_{i}}\left(\frac{\lambda^{2}\left(t-T_{0}\right)}{m_{i-1}-m_{k}+\lambda}\right)^{\alpha_{i}}\right)\left(K_{k} e^{-m_{k}\left(t-T_{0}\right)}+\frac{d_{k}}{t-T_{0}}\right) .
\end{aligned}
$$

Corollary 4. (Upper bound of the expected value) Let the conditions of Lemma 7 be satisfied and there exists positive constants $M, M_{k}, \mu, \mu_{k}: 0<$ $\mu_{k} \leq \lambda, k=0,1,2, \ldots, 0<\mu \leq \lambda$ such that for any $t \geq T_{0}$

$$
e^{-m_{0}\left(t-T_{0}\right)} \frac{\left(\lambda\left(t-T_{0}\right)\right)^{\alpha_{1}-1}}{\left(\alpha_{1}-1\right) !} \leq M_{0} e^{\mu_{0}\left(t-T_{0}\right)}
$$

and

$$
\left.\left(K_{k} e^{-m_{k}\left(t-T_{0}\right)}+\frac{d_{k}}{t-T_{0}}\right) \prod_{i=1}^{k}\left(\left|b_{i}\right| e^{\lambda d_{i}} \frac{\lambda^{2}\left(t-T_{0}\right)}{m_{i-1}-m_{k}+\lambda}\right)^{\alpha_{i}}\right) \leq M_{k} e^{\mu_{k}\left(t-T_{0}\right)}
$$

with

$$
\sum_{k=0}^{\infty} M_{k} e^{\mu_{k}\left(t-T_{0}\right)} \leq M e^{\mu\left(t-T_{0}\right)}
$$

Then

$$
E\left(\left|u\left(t ; T_{0}, u_{0},\left\{\tau_{k}\right\}\right)\right|\right) \leq M\left|u_{0}\right| e^{-(\lambda-\mu)\left(t-T_{0}\right)}
$$


Corollary 5. Let the conditions of Lemma 7 with $m_{k}=m$. Then

$$
\begin{gathered}
E\left(\left|u\left(t ; T_{0}, u_{0},\left\{\tau_{k}\right\}\right)\right|\right) \leq\left|u_{0}\right| e^{-(m+\lambda)\left(t-T_{0}\right)}\left\{\frac{\left(\lambda\left(t-T_{0}\right)\right)^{\alpha_{1}-1}}{\left(\alpha_{1}-1\right) !}\right. \\
+\left(K_{k}+\frac{d_{k} e^{m\left(t-T_{0}\right)}}{t-T_{0}}\right) \sum_{k=1}^{\infty}\left(\prod_{i=1}^{k}\left|b_{i}\right| e^{\lambda d_{i}}\left(\lambda\left(t-T_{0}\right)\right)^{\alpha_{i}}\right) .
\end{gathered}
$$

If additionally there exist positive constants $D, \mu: \mu<\lambda+m$ such that

$$
\left(K_{k}+\frac{d_{k} e^{m\left(t-T_{0}\right)}}{t-T_{0}}\right) \sum_{k=1}^{\infty}\left(\prod_{i=1}^{k}\left|b_{i}\right| e^{\lambda d_{i}}\left(\lambda\left(t-T_{0}\right)\right)^{\alpha_{i}}\right) \leq D e^{\mu\left(t-T_{0}\right)}
$$

then

$$
E\left(\left|u\left(t ; T_{0}, u_{0},\left\{\tau_{k}\right\}\right)\right|\right) \leq M\left|u_{0}\right| e^{-\nu\left(t-T_{0}\right)}
$$

where $\nu=\min \{m, m+\lambda-\mu\}$ and $M=1+D$.

Proof. From(26) using $\frac{\left(\lambda\left(t-T_{0}\right)\right)^{\alpha_{1}-1}}{\left(\alpha_{1}-1\right) !} \leq e^{\lambda\left(t-T_{0}\right)}$ we obtain

$$
E\left(\left|u\left(t ; T_{0}, u_{0},\left\{\tau_{k}\right\}\right)\right|\right) \leq\left|u_{0}\right| e^{-(m+\lambda)\left(t-T_{0}\right)}\left(e^{\lambda\left(t-T_{0}\right)}+D e^{\mu\left(t-T_{0}\right)}\right) .
$$

Remark 5. Note inequality (27) is satisfied for $\alpha_{k} \leq H, d_{k} \in(0, d]$, and $b_{i}:\left|b_{k}\right| \leq e^{-\lambda d_{k}} s^{\alpha_{k}} \frac{A_{k-1} !}{A_{k} !}, k=1,2, \ldots$, where $s \in(0,1), H, d$ are positive 
constants. Indeed,

$$
\begin{aligned}
& \sum_{k=1}^{\infty}\left(\prod_{i=1}^{k}\left|b_{i}\right| e^{\lambda d_{i}}\left(\lambda\left(t-T_{0}\right)\right)^{\alpha_{i}}\right)\left(K_{k}+\frac{d_{k} e^{m\left(t-T_{0}\right)}}{t-T_{0}}\right) \\
& \leq \sum_{k=1}^{\infty}\left(\prod_{i=1}^{k} s^{\alpha_{i}} \frac{A_{i-1} !}{A_{i} !}\left(\lambda\left(t-T_{0}\right)\right)^{\alpha_{i}}\right)\left(1+\lambda\left(t-T_{0}\right)^{\alpha_{k+1}-1}\left(\alpha_{k+1}-1\right)\right. \\
& \left.\quad+d_{k} \frac{e^{m\left(t-T_{0}\right)}}{t-T_{0}}\right) \\
& \leq \sum_{k=1}^{\infty} s \lambda \frac{\left(s \lambda\left(t-T_{0}\right)\right)^{A_{k}}}{A_{k} !}\left(\left(t-T_{0}\right)+\lambda\left(\alpha_{k+1}-1\right)\left(t-T_{0}\right)^{\alpha_{k+1}}\right. \\
& \left.+d e^{m\left(t-T_{0}\right)}\right) \\
& \leq s \lambda \sum_{k=1}^{\infty} \frac{\left(s \lambda\left(t-T_{0}\right)\right)^{A_{k}}}{A_{k} !}\left(L e^{m\left(t-T_{0}\right)}+d e^{m\left(t-T_{0}\right)}\right) \\
& \leq s \lambda(L+d) e^{m\left(t-T_{0}\right)} \sum_{k=1}^{\infty} \frac{\left(s \lambda\left(t-T_{0}\right)\right)^{A_{k}}}{A_{k} !} \leq s \lambda(L+d) e^{(m+s \lambda)\left(t-T_{0}\right)} .
\end{aligned}
$$

\section{5. p-Moment Exponential Stability for RNIDE}

The main goal of the paper is to define the exponential stability of the zero solution of RNIDE (6) (with $x_{0}=0$ ) and to obtain some sufficient conditions for it.

Definition 3. Let $p>0$. Then the trivial solution $\left(x_{0}=0\right)$ of the RNIDE (6) is said to be p-moment exponentially stable if for any initial point $x_{0} \in \mathbb{R}^{n}$ there exist constants $\alpha, \mu>0$ such that the inequality $\left.E\left[\| x\left(t ; T_{0}, x_{0},\left\{\tau_{k}\right)\right\}\right) \|^{p}\right]<$ $\alpha\left\|x_{0}\right\|^{p} e^{-\mu\left(t-T_{0}\right)}$ holds for all $t \geq T_{0}$, where $x\left(t ; T_{0}, x_{0},\left\{\tau_{k}\right)\right\}$ is the solution of the IVP for the RNIDE (6).

Remark 6. We note that the two-moment exponentially stability for stochastic equations is known as exponentially stability in mean square.

Definition 4. Let $J \subset \mathbb{R}_{+}$be a given interval and $\Delta \subset \mathbb{R}^{n}, \quad 0 \in \Delta$ be a given set. We will say that the function $V(t, x): J \times \Delta \rightarrow \mathbb{R}_{+}, V(t, 0) \equiv 0$ 
belongs to the class $\Lambda(J, \Delta)$ if it is continuous on $J \times \Delta$ and locally Lipschitzian with respect to its second argument.

For functions $V(t, x) \in \Lambda(J, \Delta)$ we will use Dini derivatives defined by:

$$
D_{+} V(t, x)=\limsup _{h \rightarrow 0^{+}} \frac{1}{h}\{V(t, x)-V(t-h, x-h f(t, x))\}, \quad t \in J, x \in \Delta,
$$

where there exists $h_{1}>0$ such that $t-h \in J, x-h f(t, x) \in \Delta$ for $0<h \leq h_{1}$.

Theorem 1. Let the following conditions be satisfied:

1. Conditions (H3), (H4), (H5) and one of the conditions (H1) or (H2) hold.

2. The function $V \in \Lambda\left(\left[T_{0}, \infty\right), \mathbb{R}^{n}\right)$ and there exist positive constants $a, b$ such that

(i) $\quad a\|x\|^{p} \leq V(t, x) \leq b\|x\|^{p}$ for $t \geq T_{0} x \in \mathbb{R}^{n}$;

(ii) there exists a constant $m: 0<m \leq \lambda$ such that the inequality

$$
D_{+} V(t, x) \leq-m V(t, x), \quad \text { for } t>T_{0}, x \in \mathbb{R}^{n}
$$

holds;

(iii) for any $k=1,2, \ldots$ there exist functions $w_{k} \in C\left(\mathbb{R}_{+}, \mathbb{R}_{+}\right)$such that

$$
V\left(t, I_{k}(x)\right) \leq w_{k}(t) V(t, x) \quad \text { for } t \geq T_{0}, x \in \mathbb{R}^{n} .
$$

3. There exist positive constants $D, \mu: \mu<\lambda+m$ and $C_{k}<1, k=1,2, \ldots$ such that $w_{k}(t) \leq C_{k}$ for $t \geq T_{0}$ and

$$
\sum_{k=1}^{\infty}\left(\prod_{i=1}^{k} C_{i} e^{\lambda d_{i}}\left(\lambda\left(t-T_{0}\right)\right)^{\alpha_{i}}\right)\left(K_{k}+\frac{d_{k} e^{m\left(t-T_{0}\right)}}{t-T_{0}}\right) \leq D e^{\mu\left(t-T_{0}\right)}, t \geq T_{0} .
$$

Then the trivial solution of the RNIDE (6) is p-moment exponentially stable.

Proof. Let $x_{0} \in \mathbb{R}^{n}$ be an arbitrary initial point and the stochastic process $x_{\tau}(t)=x\left(t ; T_{0}, x_{0},\left\{\tau_{k}\right)\right\}$ be a solution of the initial value problem for the RNIDE (6).

Now consider the IVP for the scalar linear RNIDE (18) with $m_{k}=m$, $b_{k}=C_{k}$ for $k=1,2, \ldots$, and $x_{0}=V\left(T_{0}, x_{0}\right)$. According to Lemma 7 the solution $u\left(t ; T_{0}, V\left(T_{0}, x_{0}\right),\left\{\tau_{k}\right\}\right)$ of RNIDE (18) is given by (19).

Let $t_{k}$ be arbitrary values of the random variables $\tau_{k}, k=1,2, \ldots$ and $T_{k}=T_{0}+\sum_{i=1}^{k} t_{i}+\sum_{i=1}^{k} d_{i}, k=1,2, \ldots$ are values of the random variables $\xi_{k}$. 
Define $v(t)=V\left(t, x\left(t ; T_{0}, x_{0},\left\{T_{k}\right\}\right)\right), t \geq T_{0}, t \neq T_{k}$ and $v\left(T_{k}\right)=V\left(T_{k}, x\left(T_{k}-\right.\right.$ $\left.0 ; T_{0}, x_{0},\left\{T_{k}\right\}\right), k=1,2, \ldots$

Let $t \in\left(T_{k}, T_{k}+d_{k+1}\right], k=0,1,2, \ldots$ Using the continuity and monotonicity of the function $V(t, x)$ and condition 2 (iii) we obtain for $t \in\left(T_{k}, T_{k}+\right.$ $\left.d_{k+1}\right], k=0,1,2, \ldots$

$$
\begin{aligned}
v(t) & =V\left(t, I_{k}\left(x\left(T_{k}-0\right)\right)\right) \\
& \leq w_{k}(t) V\left(t, x\left(T_{k}-0\right)\right) \\
& \leq w_{k}(t) V\left(T_{k}, x\left(T_{k}-0\right)\right) \\
& =w_{k}(t) v\left(T_{k}-0\right) \\
& \leq C_{k} v\left(T_{k}-0\right) .
\end{aligned}
$$

Now, consider any interval $\left(T_{k}+d_{k+1}, T_{k+1}\right]$. Then using $v\left(T_{k}+d_{k+1}\right)=$ $V\left(T_{k}+d_{k+1}, x\left(T_{k}+d_{k+1} ; T_{0}, x_{0},\left\{T_{k}\right\}\right)\right)$ we obtain

$$
\begin{aligned}
v^{\prime}(t) & =D_{+} v(t)=D_{+} V\left(t, x\left(t ; T_{0}, x_{0},\left\{T_{k}\right\}\right)\right) \\
& \leq-m V\left(t, x\left(t ; T_{0}, x_{0},\left\{T_{k}\right\}\right)\right) \\
& =-m v(t), t \in\left(T_{k}+d_{k+1}, T_{k+1}\right] .
\end{aligned}
$$

Therefore, from (30) and (31) it follows the function $v(t)$ satisfies the linear impulsive differential inequalities with fixed points of noninstantaneous impulses

$$
\begin{aligned}
& v^{\prime}(t) \leq-m v(t) \quad \text { for } \quad T_{k}+d_{k+1}<t<T_{k+1}, k=1,2, \ldots, \\
& v\left(T_{k}+\right) \leq C_{k} v\left(T_{k}\right), \quad \text { for } \quad T_{k}<t \leq T_{k}+d_{k+1}, \quad k=1,2, \ldots, \\
& v\left(T_{0}\right)=V\left(T_{0}, x_{0}\right) .
\end{aligned}
$$

Consider the function $m(t)=v(t)-u\left(t ; T_{0}, V\left(T_{0}, x_{0}\right),\left\{T_{k}\right\}\right), t \geq T_{0}$ which is piecewise continuous function and according to Proposition 1 the function $m(t)$ is nonpositive on $\left[T_{0}, \infty\right)$ i.e.

$$
v(t) \leq u\left(t ; T_{0}, x_{0},\left\{T_{k}\right\}\right) \quad \text { for } t \geq T_{0}
$$

Note inequality (33) is satisfied for any arbitrary given sequence of fixed points of impulses $\left\{T_{k}\right\}$. Therefore, the generated by $v(t)$ stochastic process $v_{\tau}(t)$ satisfies the inequality $v_{\tau}(t) \leq u\left(t ; T_{0}, x_{0},\left\{\tau_{k}\right\}\right)$.

From Corollary 5 and inequality (27) with $m_{i}=m$ and $b_{i}=C_{i}$ and condi- 
tion $2(i)$ of Theorem 1 we obtain the inequalities

$$
\begin{aligned}
E\left(\left\|x_{\tau}(t)\right\|^{p}\right) & =\frac{1}{a} E\left(a\left\|x_{\tau}(t)\right\|^{p}\right) \\
& \leq \frac{1}{a} E\left(V\left(t, x_{\tau}(t)\right)\right) \\
& \leq \frac{1}{a} E\left(v_{\tau}(t)\right) \\
& \leq \frac{1}{a} E\left(u\left(t ; T_{0}, x_{0},\left\{\tau_{k}\right\}\right)\right) \\
& \leq \frac{M}{a} V\left(T_{0}, x_{0}\right) e^{-\nu\left(t-T_{0}\right)} \\
& \leq \frac{M b}{a}\left\|x_{0}\right\|^{p} e^{-\nu\left(t-T_{0}\right)}, \quad t \geq T_{0},
\end{aligned}
$$

where $\nu=\min \{m, m+\lambda-\mu\}$ and $M=1+D$.

Inequality (34) proves the p-moment exponential stability.

\section{Acknowledgments}

Research was partially supported by the Fund Scientific Research No. MU15FM IIT008, Plovdiv University.

\section{References}

[1] R. Agarwal, D. O'Regan, S. Hristova, Stability of Caputo fractional differential equations with non-instantaneous impulses, Commun. Appl. Anal., 20 (2016), 149-176.

[2] R. Agarwal, D. O'Regan, S. Hristova, Stability by Lyapunov like functions of nonlinear differential equations with non-instantaneous impulses, J. Appl. Math. Comput., accepted.

[3] R. Agarwal, D. O'Regan, S. Hristova, Stability of Caputo fractional differential equations by Lyapunov functions, Appl. Math., 60 (2015), 653-676.

[4] A. Anguraj, A. Vinodkumar, Existence, uniqueness and stability results of random impulsive semilinear differential systems, Nonlinear Anal. Hybrid Syst., 3 (2010), 475-483.

[5] D.D. Bainov, P.S. Simeonov, Systems with Impulsive Effect. Stability, Theory and Applications, Ellis Horwood Series: Mathematics and its Applications, Ellis Horwood Ltd., Chichester Halster Press, John Wiley \&Sons, Inc., New York, 1989.

[6] D. Bainov, P. Simeonov, Impulsive Differential Equations: Periodic Solutions and Applications, Pitman Monographs and Surveys in Pure and Applied Mathematics, 66, Longman Sciencific \& Technical, Harlow; Co-published in USA, John Wiley \& Sons, Inc., New York, 1993. 
[7] J. M. Borwein, O-Y. Chan, Uniform bounds for the complementary incomplete gamma function, Math. Inequal. Appl., 12, No. 1 (2009), 115-121.

[8] K.E.M. Church, R.J. Smith, Existence and uniqueness of solutions of general impulsive extension equations with specification to linear equations, Dyn. Cont., Discr. Impuls. Syst., Ser. B: Appl., Algor., 22 (2015) 163-197.

[9] S. Das, D.N. Pandey, N. Sukavanam, Existence of solution of impulsive second order neutral integro- differential equations with state delay, J. Integ. Eq. Appl., 27, No. 4 (2015), 489-520.

[10] E. Hernandez, M. Pierri, Donal O'Regan, On abstract differential equations with non instantaneous impulses, Topological Methods in Nonl. Anal. , 46, No. 2 (2015), 10671088 .

[11] V. Lakshmikantham, D.D. Bainov, P.S. Simeonov, Theory of Impulsive Differential Equations, Series in Modern Applied Mathematics, 6, Word Scientific Publications Co., Teaneck, NJ, 1989, 273pp.

[12] P. Li, Ch. Xu, Boundary value problems of fractional order differential equation with integral boundary conditions and not instantaneous impulses, J. Function Spaces, 2015 (2015), Article ID 954925, 9 pages.

[13] Y.M. Liao, J.R. Wang, A note on stability of impulsive differential equations, Boundary Value Problems, 2014, 2014:67, 8 pages.

[14] J.M. Sanz-Serna, A.M. Stuart, Ergodicity of dissipative differential equations subject to random impulses, J. Diff. Equ., 155 (1999), 262-284.

[15] Wu S., D. Hang, X. Meng, p-Moment Stability of Stochastic Equations with Jumps, Appl. Math. Comput., 152 (2004), 505-519.

[16] Wu H., J. Sun, p-Moment Stability of Stochastic Differential Equations with I mpulsive Jump and Markovian Switching, Automatica, 42 (2006), 1753-1759.

[17] Yang J., Zhong S., Luo W., Mean square stability analysis of impulsive stochastic differential equations with delays, J. Comput. Appl. Math., 216, No. 2 (2008), 474-483.

[18] J.R. Wang, M. Feckan, Y. Zhou, Random noninstantaneous impulsive models for studying periodic evolution processes in pharmacotherapy, Math. Model. Appl. Nonlin. Dyn., Ser. Nonlinear Systems and Complexity, 14 (2016), 87-107. 